\title{
Korrespondenzen.
}

\section{Zur Richtigstellung des Briefes von Fritsch an den Herausgeber der Deutschen medizinischen Wochenschrift (No. 16).}

Von Prof. Dr. Dührsen in Berlin.

Schon vor einem Jahre habe ich an Fritsch geschrieben, daß ich mich, so weit es in meinen Kräften steht, bemühe, die Wissenschaft zu fördern, und daß es mir gleichgültig ist, ob hieraus für meine Person ein Schaden oder ein Nutzen entsteht. Nach dieser Richtung hin ist mir also die Wirkung des Gampschen Artikels gleichgültig. Ioh habe meiner Ansicht nach noch eine wissenschaftlich und sozial wichtige Aufgabe zu erfüllen, und diese Aufgabe besteht in der Einführung des vaginalen Kaiserschnitts in die allgemeine ärztliche Praxis. Jeder Arzt muß später meiner Ansicht nach imstande sein, im Notfall den vaginalen Kaiserschnitt in der Praxis auszuführen, wie er in der Praxis auch eine Zange, eine Wendung, eine Perforation zu machen hat. In der Tat läßt sich der vaginale Kaiserschnitt vom Arzt allein mit Erfolg ausführen, wenn es auch jetzt vielleicht von einem vernünftigen Arzt, der den letzten Fortschritt auf diesem Gebiete nicht verfolgt hat, nicht zugegeben wird. Die von mir bereits vor zwei Jahren angegebene Vereinfachung des vaginalen Kaiserschnitts, der sogenannte Metreurynter-Schnitt, verwandelt eben die schwere und nur von einem Frauenarzt auszuführende Operation des vaginalen Kaiserschnitts in eine verhältnismäBig einfache und jeden. falls viel leichtere Operation, als es manche vom praktischen Arzt auszuführende Zange oder Perforation ist. Selbstverständlich wird jeder praktische Arzt sich in absehbarer Zeit noch bemühen, zu einer solchen Operation einen Frauenarzte zuzuziehen oder die Gebärenden in eine Klinik zu überführen. Ist dies jedoch nicht möglich und hat sich der Arzt praktisch oder theoretisch mit meinem Metreurynter-Schnitt 
bekannt gemacht, so wird er nicht mit leichtem Herzen, aber doch mit dem ruhigen Bewußtsein die Operation ausführen, das dem Arzt die Achtung vor jedem lebenden und lebensfähigen Wesen einflößen muß. Wir müssen eben auch in Deutschland dahin kommen, wie das Pinard in Frankreich schon seit vier Jahren erstrebt, daß die Perforation eines lebenden und lebensfähigen Kindes aus der Reihe der legalen geburtshilflichen Operationen gestrichen wird. Ja wir müssen auch noch weiter kommen und müssen es verhüten, daß ein Kind, das lebensfähig und lebensfrisch in die Geburt eintritt, infolge von Geburtsstörungen zugrunde geht. Dies läßt sich in der Tat, wie Seitz mit aller Exaktheit nachgewiesen hat, durch die von mir angegebenen Methoden der Beseitigung aller Weichteilshindernisse erreichen, zuma! wenn man den vaginalen Kaiserschnitt in der von meinem Assistenten Dr. Solms angegebenen Kombination mit einem extraperitonealen Flankenschnitt auch bei engem Becken anwendet.

Die erwähnten Methoden lassen sich vollkommener noch als die übrigen geburtshilflichen Operationen einüben: Für die Einübung des Metreurynter-Schnitts habe ich ein besonderes, billiges Phantom angegeben, an dem sich im Notfalle der Arzt selbst den Metreurynter-Schnitt einüben kann. Auch die erwähnte Kombination, die ich als Laparo-KolpoHysterotomie oder Buddha-Geburt bezeichne, läßt sich in der Weise lehren, daß der Flankenschnitt im "Chirurgischen Operationskursus" eingeübt wird. Hierfür wollte ich auf dem letzten Chirurgen-Kongreß in Berlin die klinischen Lehrer der Chirurgie interessieren - leider konnte ich jedoch den Vortrag nicht halten, weil er auf die letzte Sitzung verschoben und diese durch kasuistische Mitteilungen vollständig in Anspruch genommen wurde. Immerhin hatte mir aber doch der Vorsitzende, Herr Geheimrat Bier, freundlicherweise die Gelegenheit gegeben, wenigstens eine Patientin mit ihrem Kinde zu demonstrieren, bei der ein praktischer Arzt, Herr Dr. Töpfer in Friedenau, die BuddhaGeburt mit Erfolg im Privathause ausgeführt hatte, trotzdem das enge Becken dieses Falles noch mit Placenta praevia kompliziert war.

Von all diesen meinen Bestrebungen, für welche ich auf Grund meiner wissenschaftlichen Vergangenheit eine unbefangene Prüfung verlange, scheint Herr Fritsch nichts zu wissen - jedenfalls ignoriert er sie vollständig; anderseits haben die französischen hervorragendsten Geburtshelfer diesen meinen Methoden gegenüber einen anderen Standpunkt eingenommen. Ich habe hier in Paris jetzt durch die liebenswürdige Einladung der Professoren Pinard ųnd Bar Gelegenheit gehabt, in den beiden staatlichen Kliniken dieser Herren einen klinischen Vortrag vor den Studierenden zu halten und bei dieser Gelegenheit auch die Ausführung des Metreurynter-Schnittes am Phantom zu demonstrieren, weil sich gerade keine Gelegenheit zu einer Operation an der Lebenden bot. In der Pinardschen Klinik schloß sich an meinen Vortrag eine Diskussion zwischen Pinard und mir an, in welcher Pinard hervorhob, daß mein Metreurynter-Schnitt eine technisch einfache Operation sei. In der Klinik von Paul Bar hie't diesər Ge'ehrte zunächst einen e.nleitondea Vortrag, in we'chem er mit ganz außerordentlicherS Schkonntnis und Klarheit den Studierenden die Technik und die Bedeutung des vaginalen Kaiserschnittes, des Metreurynter-Schnittes und der BuddhaGeburt auseinandersetzte. Ja, er hatte sogar zu meiner großen Ueberraschung meine sämtlichen Abbildungen, auch die neuesten in der Weise zurVerfïgung, daß er sie den Studierenden in Lichtbildern zeigen konnte. Ist dies nicht eine Bestätigung des Gampschen Artikels „Der Prophet in seinem Vaterlande"?

Es ist also durchaus keine leichtfertige Behauptung der Herren Abgeordneten Schmedding und $\mathrm{Gamp}$, daß es möglich sein wird, allein in Dautschland jährlich 50000 Kinder zu retten; viel eher ist die Behauptung, daß das nicht möglich sei, voreilig, wenn man alle die Vorschläge, welche auf die Erreichung dieses Zieles hinarbeiten, ni ht unberücksichtigt läßt. Man gebe mir doch erst mal Gelegenheit, im großen Maß. stabe zu beweisen, wie leicht diese Methoden auszuführen sind und wie leicht sie sich lehren lassen! Es hat sich in der Wissenschaft schon oft gerächt, daß man neue Methoden kurzerhand als nicht zum Ziele führend oder als unsinnig verworfen hat.

Ich komme jetzt zu meinen persönlichen Wünschen. Man könnte vielleicht annehmen, daß ich eine ordentliche Professur jetzt noch als etwas basonders für mich Erstrebenswertes, als eine Versorgung auf Lebenszeit betrachte. Dies ist nicht der Fall; ich wünsche weiter nichts, als für einige Jahre Gelegenheit zu hạben, an einem großen geburtshilflichen Material die Studierenden und die praktischen Aerzte in diesen vereinfachten Methoden auszubilden. Ich glaube, daß dies ein bescheidener Wunsch ist, dessen Erfüllung die objektiv denkende Mitwelt und jedenfalls die Nachwelt nur begreiflich finden wird.

Ich komme noch zu einigen direkten Widersprüchen des Herrn! Fritsch: Jetzt sagt er auf einmal, daß nicht der vaginale Kaisersehnitt. sondern die backenerweiternde Operation eine neue Aera der Geburtshilfe begründet habe. Da muß man wirklich an das Wort im ,Wallenstein" denken: „Vor Tisch las man's anders"! denn in der Deutschen medizinischen Wochenschrift 1908, No. 33 hat Fritsch einen Artikel veröffentlicht, betitelt ,,Alte und neue Geburtshilfe", und dort erklärt er, daß die neue Geburtshilfe auf dem vaginalen Kaisersehnitt und der beckenerweiternden Operation beruhe. Damals hat er mit Recht den vaginalen Kaiserschnitt zuerst genannt; denn die Zahl der Fälle, bei denen er in Betracht kommt, ist nach Seitz dreimal größer als die Zahl der Fälle, wo die beckenerweiternde Operation gemacht wird. Außerdem wird aber meiner Ueberzeugung nach niemand mehr die beckenerweiternde Operation machen, der einmal eine Buddha-Geburt vorgenommen hat. Auch die Methoden von Frank und Sellheim, deren hohen Wert ich durchaus zu würdigen weiß, können, was Einfachheit und Lebenssicherheit anbelangt, nicht mit der Buddha-Geburt konkurrieren. Jedenfalls ist die Buddha-Geburt die einzige Methode, die sich im Privathaus ausführen läßt. Einen äußeren extraperitonealen Schnitt empfehle ich nur bei engen Becken -- bei allen Weichteilshindernissen kommt man mit den vaginalen Schnittmethoden aus - und wenn manchen Geburtshelfern auch bei einfachen Weichteilshindernissen der äußere Schnitt lieber ist als der vaginale. so werden die Frauen selbst sicherlich nicht dieser An. sicht sein.

Dar vaginale Kaiserschnitt kommt übrigens nicht vor allem bei Eklampsie in Betracht; er kommt, wie das in der Münchener Klinik ja mit den besten Erfolgen in die Wirklichkeit umgesetzt ist, in all den Fällen in Betracht, wo Lebensgefahr für die Mutter oder das Kind auftritt und bei mangelhaft erweitertem Muttermund mildere Dilatations-Methoden, vor allem die Metreuryse nicht zum Ziele führen. Bei dieser Ausdehnung des vaginalen Kaiserschnitts läßt sich die Zahl der geburtshilflichen Operationen, wie das ebenfalls aus der Doederleinschen Klinik nachgewiesen ist, sogar verringern. Man komme mir also nicht etwa noch mit dem Schlagwort „Furor operativus“, das mir gegenüber schon oft an. gewandt ist.

Dəm gegenüber kann ich mit einem anderen Wort aufwarten, das von einem der ersten französischen Chirurgen bzw. Gynäkologen stammt (Pozzi), der mit mir jetzt als Ehrengast zu der Centenarfeier nach Buenos-Aires reist. Pozzi hat den vaginalen Kaisersehnitt als die letzte chirurgische Großtat des verflossenen Jahrhunderts bezeichnet, und der angesehene amerikanische Gynäkologe Reuben Peterson hat, um nur noch eine Stimme anzuführen, mich auf Grund der Erfindung des vaginalen Kaiserschnitts jeglicher Ehrung für würdig erklärt. Uebrigens hat Peterson die Einführung meines vaginalen Kaiserschnitts, und zwar sogar in seiner ursprünglichen Form, in die allgemeine ärztliche Praxis für durchaus möglich und wünchenswert erklärt. Man sieht also, daß meine Wünsche durchaus nicht etwa Utopien sind.

Was die Besetzung der neun geburtshilflichen Lehrstühle in Preußen anbelangt, so lehren Fritsch in Bonn, K üstner in Breslau, Jung in Göttingen, Krömer in Greifswald, "Veit in Halle, Franz in Kiel, Winter in Königsberg und Stöckl in Marburg. Von diesen Herren sind Fritsch, Küstner und Winter frühere Assistenten von Olshausen, und Stöckl ist der Schwiegersohn von Fritsch. Jung ist ein früherer Assistent von Martin, der mit Olshausen zusammen viele Jahre lang die geburtshilfliche Gesellschaft geleitet hat. Veit hat mit Olshausen zusammen das Schrödersche Lehrbuch herausgegeben, und Franz ist der Schwiegersohn des eng nit Fritsch befreundeten Herrn Fehling in Straßburg. Krö mer ist ein Schüler von Bum $\mathrm{m}$, und Bum $\mathrm{m}$ hat doch jedenfalls unter Mitwirkung von Olshausen seine Berliner Stellung bekommen. Es ist daher doch wohl nicht ernstlich zu bestreiten, daß alle preußischen Universitäten mit Assistenten resp. Freunden von Olshausen besetzt sind, die nicht gegen den Wunsch von Olshausen einen wissenschaftlichen Gegner Olshausens für eine ordentliche Professur empfehlen werden. Den besten Beweis hierfür hat ja Fritsch selbst geliefert, indem er mir vor einem Jahr schrieb: ,Ich bedaure sehr, $\mathrm{da} ß$ Sie sich durch Ihren Streit mit Ohlshausen Ihre akademische Laufbahn verdorben haben!" Man hat allerdings noch andere persönlich gegen mich sprechende Gründe an maßgebender Stelle vorgebracht, die jedoch alle auf teils lächerlichen, teils infamen Behauptungen beruhen, deren Unwahrheit ich dokumentarisch beweisen kann.

Bezüglich meines Prozesses hat Fritsch mehrere absolut unrichtige Behauptungen aufgestellt: $\mathrm{Zu}$ der Operation hatte die Patientin nicht nur ihre Einwilligung gegeben, sondern sie hatte mich sogar durch den behandelnden Frauenarzt, einen früheren Assistenten von mir, dringend um die Ausführung der Operation gebeten und zu diesem Zwecke ihr Alter sogar statt auf 45 auf 42 Jahre angegeben. Es handelte sich in diesem Falle um eine fixierte Retroflexion, und die Patientin wünschte Konzeption. Da ich bei mehreren ebenso alten Frauen nach Vaginifixur Schwangerschaft hatte eintreten sehen, so war die Operation nach meiner wissenschaftlichen Ueberzeugung und Erfahrung durchaus berechtigt, und es war eben eine unerhörte Vergewaltigung meiner wissenschaftlichen Ueberzeugung und Erfahrung, daß Olshausen in seinem Gutachten behauptete, die Vaginifixur hätte wegen der Fixation des Uterus und wegen des Alters der Patientin keine Aussicht auf Erfolg gehabt, wäre daher nicht berechtigt und mein Vorgehen daher ein Kunstfehler gewesen. Wegen dieses Versuchs, die wissenschaftlichen, auf Erfahrung beruhenden Ueberzeugungen eines Forschers vor den Strafrichter zu bringen, war

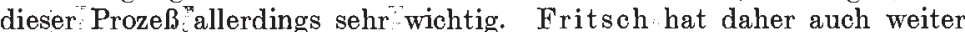
nichts gesagt, als was durchaus selbstverständlich war: daß ein Operateur berechtigt sei, eine Operation auszuführen, wenn er auf Grund seiner Er. 
fahrungen die wissenschaftliche Ueberzeugung gewonnen habe, daß die Operation den beabsichtigten Zweck erreichen könne. Für meine Freisprechung war maßgebend die Achtung der Richter vor der wissenschaft. lichen Ueberzeugung eines Operateurs, den das Urteil auf diesem Gebiete selbst als Autorität bezeichnete.

Falls ich auf etwaige weitere Angriffe in den nächsten Monaten nicht antworte, so beruht das darauf, daß ich von meiner Reise esst im Juli wieder zurück sein kann 